\title{
PerFECT 2.0: A Web-Based Platform Designed to Facilitate and Support the Diagnosis of Patients with Idiopathic Pulmonary Fibrosis in Italy
}

\author{
Carlo Vancheri (D) - Monica Bengus · Laura Bianchino · \\ Maria G. Cagnazzo • Alessandra Ghirardini · Donato Lacedonia • \\ Mercedes Pasquali · Gaetano Rea · Sonja A. Rohner · Alessandro Sanduzzi • \\ Sebastiano E. Torrisi · Alberto Pesci
}

Received: February 12, 2021 / Accepted: March 19, 2021 / Published online: May 11, 2021

(C) The Author(s) 2021

\section{ABSTRACT}

Introduction: Timely and accurate diagnosis of idiopathic pulmonary fibrosis (IPF) is challenging, requiring specific tests including chest high-resolution computed tomography (HRCT), and limited by access to specialist centres with a multidisciplinary team (MDT). Here we describe PerFECT 2.0, an Italian web-based platform designed to create a network between tertiary centres with an MDT (hubs) and secondary

Supplementary Information The online version contains supplementary material available at https:// doi.org/10.1007/s41030-021-00155-8.

C. Vancheri $(\bowtie) \cdot$ S. E. Torrisi

Department of Clinical and Experimental Medicine, Regional Referral Centre for Rare Lung Diseases, University Hospital 'Policlinico G. Rodolico-San Marco', University of Catania, via Santa Sofia 78, 95123 Catania, Italy

e-mail: vancheri@unict.it

M. Bengus · S. A. Rohner

F. Hoffmann-La Roche Ltd., Basel, Switzerland

L. Bianchino · A. Ghirardini

Roche S.p.A., Monza, Italy

M. G. Cagnazzo

Departmental Unit of Territorial Pulmonology,

Local Health Service, Lecce, Italy

D. Lacedonia

Department of Medical and Surgical Sciences,

University of Foggia, Foggia, Italy centres (spokes), aiming to facilitate the diagnosis of IPF.

Methods: PerFECT 2.0 went live on 1 November 2016. Spoke centres submit anonymised documentation (HRCT images, pathological samples, clinical data) for a second opinion on the potential diagnosis of IPF from a hub centre. HRCT images are quickly uploaded, with patientidentifying information automatically removed. The hub centre views documentation online (no downloads allowed), makes any further information requests, then returns their second opinion as free text. An e-learning area contains educational material and simulated training clinical cases. Metrics were collected for

\section{Pasquali}

Division of Pneumology, S. Corona Hospital,

Pietra Ligure, Italy

G. Rea

Department of Radiology, Monaldi Hospital, AO dei Colli, Naples, Italy

\section{A. Sanduzzi}

UNESCO Chair for Health Education and

Sustainable Development, Respiratory Division, Federico II University, Naples, Italy

A. Pesci

Università degli Studi di Milano Bicocca, ASST Monza, Monza, Italy 
2017-2019; a user survey was conducted from 30 June-31 July 2020.

Results: Ten hub centres and 137 spoke centres have registered. The requests for a second opinion numbered 251 in 2017, 270 in 2018 and 265 in 2019 (overall mean 19.9 requests per month). The proportion of requests answered was $100.0 \%$ (251) in 2017, $100.0 \%$ (270) in 2018 and $97.7 \%$ (259) in 2019. The mean response time was 15.7 days. In the user survey, of nine hub responders and 19 spoke responders, $78 \%$ and $74 \%$, respectively, reported that the platform is easy to use, and $100 \%$ and $89 \%$, respectively, would recommend the platform to colleagues.

Conclusion: The PerFECT 2.0 web-based platform has created a network that enables secondary centres to gain quick and easy access to a second opinion from a tertiary centre with an MDT through online evaluation of anonymised documentation, thereby facilitating and supporting the timely and accurate diagnosis of IPF.

Keywords: Diagnosis; E-learning; Highresolution computed tomography; Idiopathic pulmonary fibrosis; Web-based platform

\section{Key Summary Points}

Why carry out this study?

Idiopathic pulmonary fibrosis (IPF) is a rare, progressive interstitial lung disease for which diagnosis is challenging, requiring specific tests including lung imaging by high-resolution computed tomography (HRCT), and best made by a multidisciplinary team (MDT).

PerFECT 2.0 is an Italian web-based platform designed to create a network that enables secondary centres (spokes) to submit anonymised documentation (HRCT images, pathological samples and clinical data) to obtain a second opinion on the potential diagnosis of IPF from tertiary centres with an MDT (hubs).

\section{What was learned from the study?}

Since going live in Italy on 1 November 2016, 10 hub centres and 137 spoke centres have registered, with a total of 786 requests submitted by spoke centres over the period 2017-2019, and return of a second opinion by hub centres in $99 \%$ of cases, with a mean response time of 15.7 days.

In a user survey, the majority of spoke centre clinicians $(N=19)$ reported that the platform is easy to use and highly useful, and the majority of hub centre clinicians $(N=9)$ reported that the platform is easy to use.

The PerFECT 2.0 web-based platform has therefore created a network that enables secondary centres to gain quick and easy access to a second opinion from a tertiary centre with an MDT, thereby facilitating and supporting the early and accurate diagnosis of IPF.

\section{DIGITAL FEATURES}

This article is published with digital features, including a summary slide, to facilitate understanding of the article. To view digital features for this article go to https://doi.org/10.6084/ m9.figshare.14236799.

\section{INTRODUCTION}

Idiopathic pulmonary fibrosis (IPF) is a rare, progressive and ultimately fatal form of interstitial lung disease (ILD) [1-3]. Although the disease trajectory can vary, patients experience a decline in lung function over time and report worsening cough, dyspnoea and exercise limitation $[1,2]$. There are two antifibrotic medications approved for the treatment of IPFpirfenidone and nintedanib [4-7]. Both antifibrotic medications have been shown to reduce the rate of lung function decline versus placebo; however, neither can reverse existing fibrotic 
damage [8-10]. Therefore, early and accurate diagnosis of IPF and subsequent initiation of treatment to slow further irreversible fibrotic damage are of key relevance [11].

The importance of early diagnosis of IPF was demonstrated in a recent observational study of disease behaviour in patients with IPF and nonIPF ILD during the time directly before and after diagnosis (peri-diagnostic period) $[12,13]$. In this study, a greater semi-annual decline in forced vital capacity was estimated for patients with IPF versus non-IPF ILD during the peridiagnostic period, illustrating the particularly progressive nature of IPF. However, many patients with IPF report delays in diagnosis, with one survey of 45 patients with IPF finding that $87 \%$ waited over 1 year to receive a diagnosis [14]. These delays may be introduced prior to evaluation at a tertiary centre; one prospective cohort study of 129 patients with IPF reported a median of 2.2 years (interquartile range 1.0-3.8 years) between onset of dyspnoea and the initial visit to a tertiary centre [15]. Reasons for delayed specialist evaluation can include a lack of awareness of IPF in primary care or at referral centres, as well as geographical barriers or waiting lists $[16,17]$. One reason why specialist evaluation is so important is that specialist multidisciplinary teams (MDTs) have been shown to increase diagnostic accuracy in ILDs [18]; also, the 2018 IPF diagnostic guidelines recommend that multidisciplinary discussions should form part of diagnostic decisionmaking [2]. There is, therefore, a clear need to address the multifactorial barriers to early and accurate diagnosis of IPF, including the limited access to specialist centres with an MDT [16].

Here we describe Pulmonary Fibrosis Enhancing Cooperative Task (PerFECT) 2.0, an Italian web-based platform designed to create a network between specialist tertiary centres with an MDT (hubs) and less experienced secondary centres (spokes), to facilitate and support the timely and accurate diagnosis of IPF.

\section{METHODS}

\section{Set-Up and Purpose of the Platform}

PerFECT 2.0 is an Italian web-based platform sponsored by Roche S.p.A. (Monza, Italy) that went live on 1 November 2016. The platform was developed with the support of pulmonary fibrosis therapy area experts, including pulmonologists and radiologists, and was built upon an earlier prototype, PerFECT 1.0, with the key update being to shorten the time required to upload high-resolution computed tomography (HRCT) images.

Two types of user profiles can access the platform: 'hub', comprising a tertiary centre, with a higher level of diagnostic ILD expertise and an MDT available; and 'spoke', comprising a secondary centre. Hub centres and spoke centres are organised in a network fashion, with several spokes connected to a single hub, and with the connections determined by taking into consideration existing networks and logistical issues.

The primary purpose of the platform is to allow spoke centres to virtually submit clinical cases to their hub centre for evaluation of the documentation (HRCT images, pathological samples and clinical data) and return of a second opinion on the potential diagnosis of IPF. A cases area accessible from the online dashboard is used for this purpose. A separate e-learning area provides educational material, including relevant publications and a case library, populated with interesting and complex cases that are adapted for use as simulated training clinical cases.

\section{Technology and Data Protection}

The PerFECT 2.0 platform was built and is managed by DOT Tech srl (Legnano, Italy), a third-party provider, and is made available to users over the internet, a distribution model known as 'software as a service'. No download of custom software or plug-in is required. Clinicians use unique individual login credentials and have access to the online dashboard for their hub or spoke centre. The patient name is visible only to the spoke centre-the name is otherwise encrypted within the database. All 
documentation is viewed by the hub centre on the platform (with no possibility to download anything), and is automatically erased 3 months after the second opinion from a hub centre has been released. Upload of an HRCT file usually completes in approximately 2 minutes, and a video tutorial on how to do this is available at the time of upload. The hub centre views the HRCT image only on the platform, with features allowing zooming in/out, measurement and annotation (i.e. features similar to those used in normal practice).

A terms and conditions section on the platform explains how the service is provided, responsibilities and procedures. The platform is constantly supervised (upon upgrades or updates) by a third-party company (external Data Protection Officer) to ensure compliance with European Union Regulation 2016/679 (General Data Protection Regulation) and The Italian Data Protection Authority (for processing of sensitive data). No ethical approval was required. Roche S.p.A. does not have access to personal information collected by the platform and only receives operational metrics data on the platform's use. Further details regarding the technology and data protection in PerFECT 2.0 are reported in the Methods section of the electronic supplementary material.

\section{Training on How to Use the Platform}

Training on how to use the platform, along with support sessions, was provided directly by DOT Tech srl. For full details regarding the training and support given to hub and spoke centres, see the Methods section of the electronic supplementary material.

\section{Requesting a Second Opinion}

For a spoke centre, the cases area of the online dashboard displays a list of the cases submitted for a second opinion, with the status (answered or unanswered) and types of expertise requested (pulmonology, radiology and/or pathology), and with the second opinions received (see Fig. S1 in the electronic supplementary material for details).
A new request for a second opinion is created by selecting 'Create a New Case' on the dashboard and completing an online form. When uploading a new case, the clinician is prompted to provide the patient's name, sex and date of birth, and to specify the type of expertise required from the hub centre as pulmonology, radiology and/or pathology (more than one type can be requested). The clinician answers a series of questions to provide the patient history and clinical data, with some questions mandatory and others optional (Table 1). They are then prompted to attach HRCT files from their desktop (with a video tutorial provided on how to do this) and, if required, the platform provides clinicians with detailed instructions on how to ship pathological samples to hub centres for a second opinion.

Once satisfied that the online request is complete, the clinician confirms to start uploading the case to the platform. Upon successful upload, the platform confirms that the case has been sent to the hub centre. Additional information regarding requesting a second opinion is detailed in the Methods section of the electronic supplementary material.

\section{Returning a Second Opinion}

For a hub centre, clinicians receive an automatic mail alert from the platform when a second opinion has been requested. The cases area of the online dashboard displays a list of the cases received for second opinion, again with the status and the types of expertise requested, and with the second opinions given (see Fig. S1 in the electronic supplementary material for details).

Within each case, the hub centre can view anonymised documentation provided by the spoke centre, including the HRCT images, pathological samples and clinical data. Should there be any issues with the documentation provided or further information required, the hub centre notifies the spoke centre by using the 'Report a Problem' button. Once a case is viewed and the hub centre is ready to provide a response, they use the 'Second Opinion' button. The response is written within a free text box, then submitted to the spoke centre. The hub 
Table 1 Patient history and clinical data requested by the PerFECT 2.0 platform when clinicians are requesting a second opinion

\begin{tabular}{|c|c|c|c|}
\hline \multirow{2}{*}{\multicolumn{2}{|c|}{$\begin{array}{l}\text { PerFECT } 2.0 \text { platform when clinicians are requesting a } \\
\text { second opinion }\end{array}$}} & \\
\hline & & Question & Responses \\
\hline Question & Responses & \multirow{2}{*}{$\begin{array}{l}\text { History of environmental/professional } \\
\text { exposure: chemicals }\end{array}$} & \multirow{2}{*}{$\begin{array}{l}\text { Yes } \\
\text { [number of years] }\end{array}$} \\
\hline \multicolumn{2}{|l|}{ Patient data } & & \\
\hline \multirow{2}{*}{$\begin{array}{l}\text { First name } \\
\text { Surname }^{\mathrm{a}}\end{array}$} & [text value] & \multirow{3}{*}{$\begin{array}{l}\text { History of environmental/professional } \\
\text { exposure: gas, fumes, vapours }\end{array}$} & No \\
\hline & [text value] & & Yes \\
\hline Sex & Male & & $\begin{array}{l}\text { [number of years] } \\
\text { No }\end{array}$ \\
\hline Age & $\begin{array}{l}\text { Female } \\
\text { [text value }]\end{array}$ & History of exposure to birds & $\begin{array}{l}\text { Yes } \\
\text { [number of years] }\end{array}$ \\
\hline \multicolumn{2}{|l|}{ Patient history } & \multirow{3}{*}{ History of exposure to farm animals } & No \\
\hline \multirow{2}{*}{$\begin{array}{l}\text { Family history of } \\
\text { pulmonary fibrosis of any } \\
\text { cause }\end{array}$} & Yes & & Yes \\
\hline & No & & [number of years] \\
\hline \multirow[t]{3}{*}{ Smoking } & Current & & No \\
\hline & Previous & \multirow[t]{2}{*}{ History of exposure to live moulds } & Yes \\
\hline & No & & [number of years] \\
\hline Number of cigarettes per day & [number value] & & No \\
\hline Number of years as a smoker & [number value] & Use of drugs with a possible fibrosing & Yes \\
\hline \multirow[t]{3}{*}{ History of CTD: Raynaud } & Yes & effect & Past \\
\hline & [number of years] & & Current \\
\hline & No & & No \\
\hline \multirow{17}{*}{$\begin{array}{l}\text { History of CTD: } \\
\text { autoantibodies }\end{array}$} & Yes & Physical examination & \\
\hline & [number of years] & Date & [date value] \\
\hline & ANA (IIF) 1 [number value] & \multirow[t]{3}{*}{ Dyspnoea } & No \\
\hline & \multirow{2}{*}{$\begin{array}{l}\text { Rheumatoid factor [number } \\
\text { value] }\end{array}$} & & Yes, at rest \\
\hline & & & Yes, on effort only \\
\hline & ACPA [number value $]$ & \multirow[t]{7}{*}{ Cough } & No \\
\hline & Anti-Ro/SSA [number value] & & Continuous dry \\
\hline & Anti-La-SSB [number value] & & cough \\
\hline & Anti Scl70 [number value] & & Sporadic dry cough \\
\hline & Anti Jo1 [number value] & & Continuous \\
\hline & Anti-Sm [number value] & & productive cough \\
\hline & Anti-RnP [number] & & Sporadic productive \\
\hline & Anti $\mathrm{Pm} / \mathrm{Scl}$ [number value] & \multirow{3}{*}{ Velcro crackles } & \\
\hline & Ds-DNA [number value] & & Yes \\
\hline & cANCA 1 [number value] & & No \\
\hline & pANCA 1 [number value] & \multirow{2}{*}{ Cyanosis } & Yes \\
\hline & No & & No \\
\hline
\end{tabular}

Table 1 continued 
Table 1 continued

\begin{tabular}{|c|c|}
\hline Question & Responses \\
\hline \multirow[t]{2}{*}{ Finger clubbing } & Yes \\
\hline & No \\
\hline \multicolumn{2}{|l|}{ Pulmonary function } \\
\hline FVC (percent predicted/litres) & [number value] \\
\hline $\mathrm{FEV}_{1}$ (percent predicted/litres) & [number value] \\
\hline $\mathrm{FEV}_{1} / \mathrm{FVC}$ ratio & [number value] \\
\hline DLco (percent predicted) & [number value] \\
\hline Oximetry (oxygen saturation $\left[\mathrm{SpO}_{2}\right], \%$ ) & [number value] \\
\hline \multicolumn{2}{|l|}{ Six-minute walk distance } \\
\hline Distance until the first stop, $\mathrm{m}$ & [number value] \\
\hline Minutes until the first stop & [number value] \\
\hline Total distance, $\mathrm{m}$ & [number value] \\
\hline \multicolumn{2}{|l|}{ Haematochemical exams } \\
\hline Date & [date value] \\
\hline WBC, cells/mL & [data value] \\
\hline $\mathrm{RBC}$, cells $\times 10^{6} / \mathrm{mL}$ & [number value] \\
\hline Haematocrit, \% & [number value] \\
\hline Haemoglobin, g/L & [number value] \\
\hline Platelets, cells $\times 10^{3} / \mathrm{mL}$ & [number value] \\
\hline
\end{tabular}

Mandatory fields are shaded in grey

$A C P A$ anti-citrullinated protein antibodies, $A N A$ antinuclear antibody, $c A N C A$ cytoplasmic anti-neutrophil cytoplasmic antibodies, $C T D$ connective tissue disease, $D L c o$ diffusing capacity for carbon monoxide, DNA deoxyribonucleic acid, $d s$ double-stranded, $F E V_{1}$ forced expiratory volume in $1 \mathrm{~s}, F V C$ forced vital capacity, IIF indirect immunofluorescence test, $p A N C A$ perinuclear anti-neutrophil cytoplasmic antibodies, $R B C$ red blood cell, $R n P$ ribonucleoprotein, $S c l$ scleroderma, $S m$ Smith antigen, $S S A$ Sjögren's-syndrome-related antigen A, SSB Sjögren'ssyndrome-related antigen $\mathrm{B}, W B C$ white blood cell

${ }^{a}$ Information visible only to the spoke centre

centre also has the option to exit the case without taking any action at that stage. If a hub centre does not respond to a case within 2 weeks, the case could be reassigned manually to another hub; of note, partially answered cases cannot be reassigned.

After receiving a second opinion from a hub centre, the treating clinician at the spoke centre remains responsible for the patient's diagnosis and management.

\section{Platform Metrics}

Metrics were collected annually for 2017, 2018 and 2019, and included registered spoke centres and hub centres; number of requests (total, by hub/spoke, by month); types of expertise requested; resolution status; and time to return the second opinion.

\section{Platform User Survey}

Between 30 June and 31 July 2020, a newsletter distributed by DOT Tech srl invited users to participate in a survey to collect feedback, with separate sets of questions created for hub and spoke centre users. Participation in the survey was reinforced by Roche affiliate personnel and one additional reminder to participate was issued by DOT Tech srl.

\section{RESULTS}

\section{Platform Metrics}

Since going live on 1 November 2016, a total of 10 hub centres and 137 spoke centres have registered on the platform (Fig. 1). The number of spokes assigned to a hub ranged from 5 to 32 in 2017, from 6 to 32 in 2018 and from 6 to 35 in 2019. These numbers changed from year to year due to spoke centres enrolling in or discontinuing use of the platform, or transferring from one hub centre to another.

The total number of requests for a second opinion made by spoke centres was 251 in 2017, 270 in 2018 and 265 in 2019. The mean number of requests per month, calculated from January 2017 to December 2019, was 19.9 (Fig. 2). The most frequent type of expertise requested was radiology, followed by pulmonology, then pathology, with many spoke centres requesting more than one type of expertise (Fig. 3). Seventyone spoke centres sent at least one request. Fortyone spoke centres sent at least three requests in any one year, and from these active spoke 


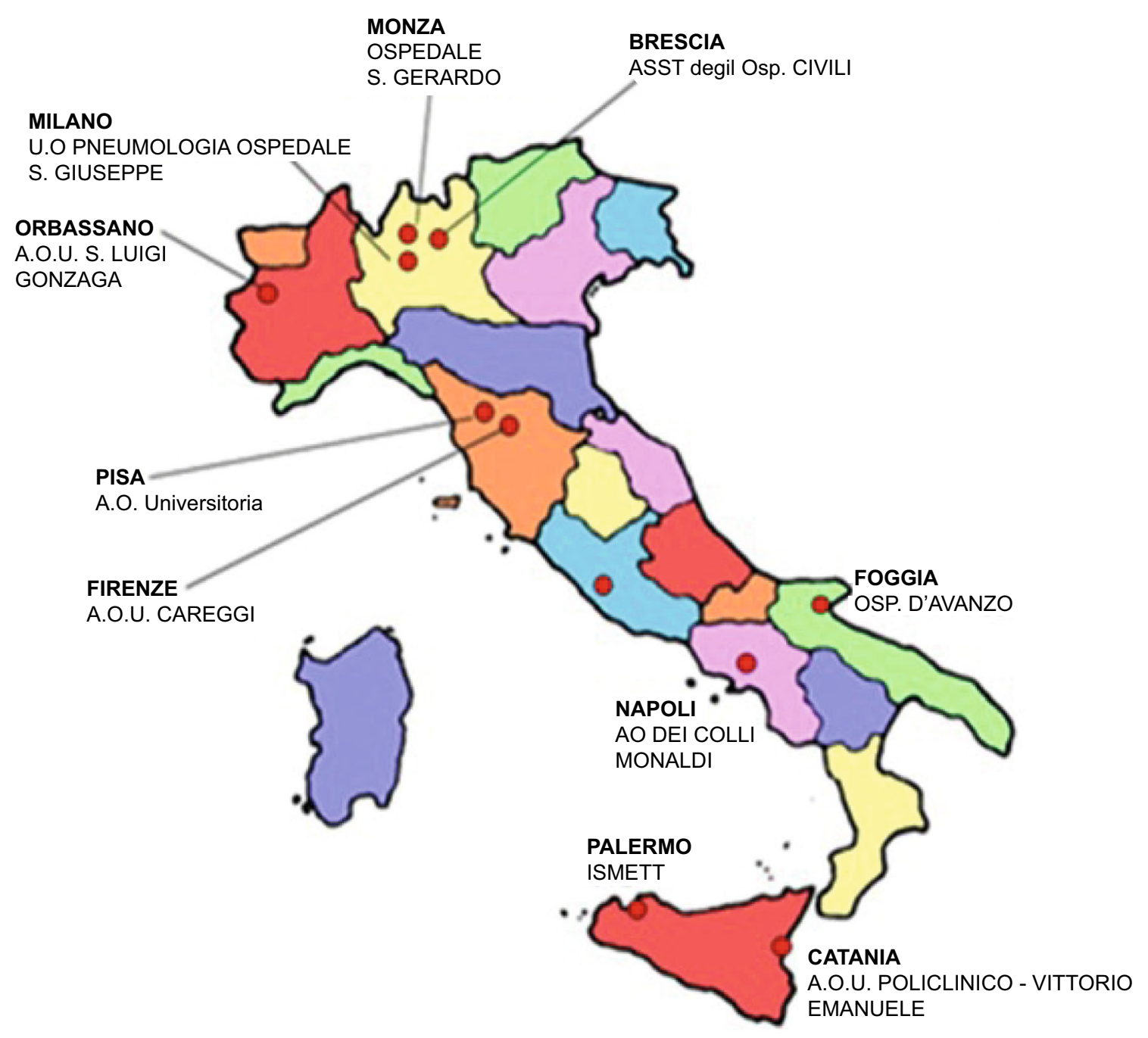

Fig. 1 PerFECT 2.0 hub and spoke centres

centres, the number of requests made ranged from 3 to 26 (total 224 from 21 centres) in 2017, from 3 to 31 (total 247 from 27 centres) in 2018 and from 3 to 25 (total 236 from 26 centres) in 2019. Around three-fifths $(61.1 \% ; 432 / 707)$ of requests from these active spoke centres originated from southern regions, with $18.5 \%$ (131) from the northwest regions, $8.6 \%$ (61) from the northeast regions, $1.3 \%$ (9) from the central regions and $10.5 \%$ (74) from the islands of Sardinia or Sicily.

The proportion of requests for a second opinion answered by hub centres was $100.0 \%$ $(251 / 251)$ in $2017,100.0 \%(270 / 270)$ in 2018 and $97.7 \%(259 / 265)$ in 2019 . The mean time for a second opinion request to be fulfilled, calculated for all hub centres from January 2017 to December 2019, was 15.7 days. The mean response times varied between the hub centres, ranging from 5.0 to 41.6 days in 2017 , from 4.7 to 30.4 days in 2018 and from 2.6 to 26.5 days in 2019. Further details regarding the platform metrics are reported in the Results section of the electronic supplementary material.

\section{User Survey Findings}

A total of 248 platform users were reached by the user survey participation newsletter, and nine clinicians from hub centres and 19 


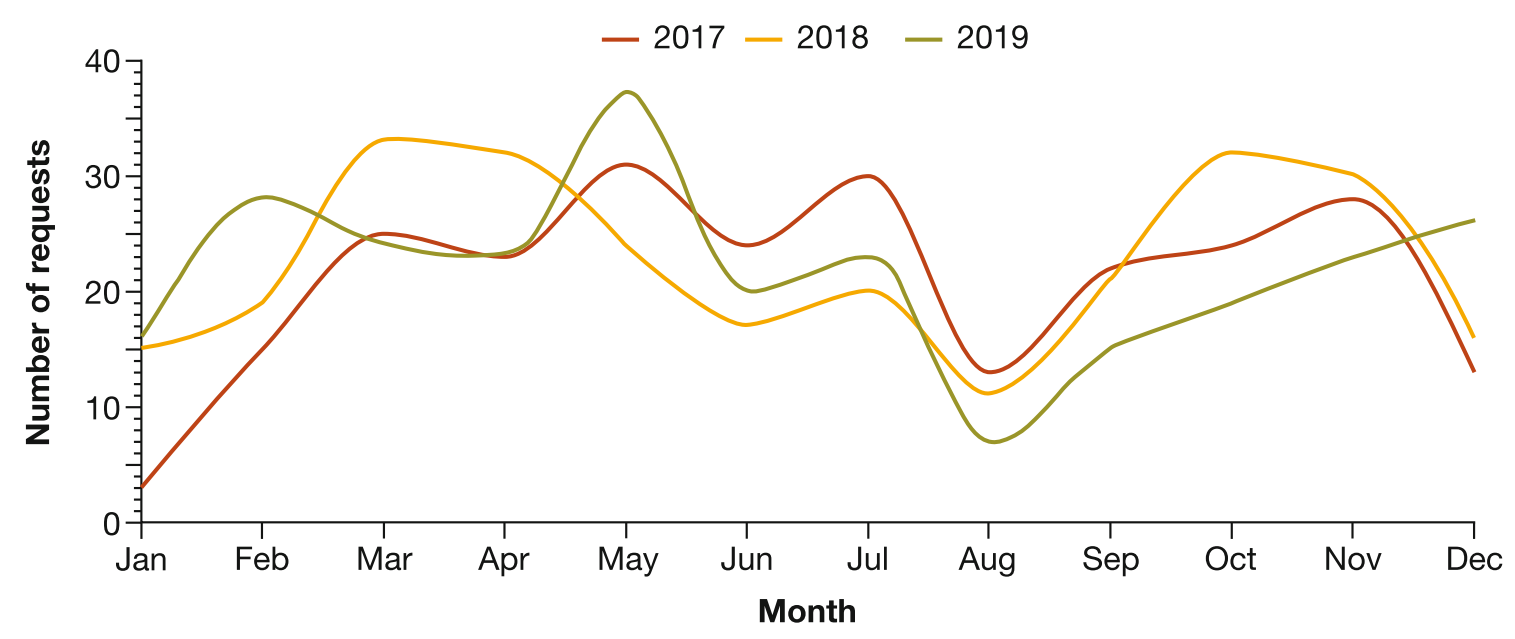

Fig. 2 Number of requests for a second opinion presented over time

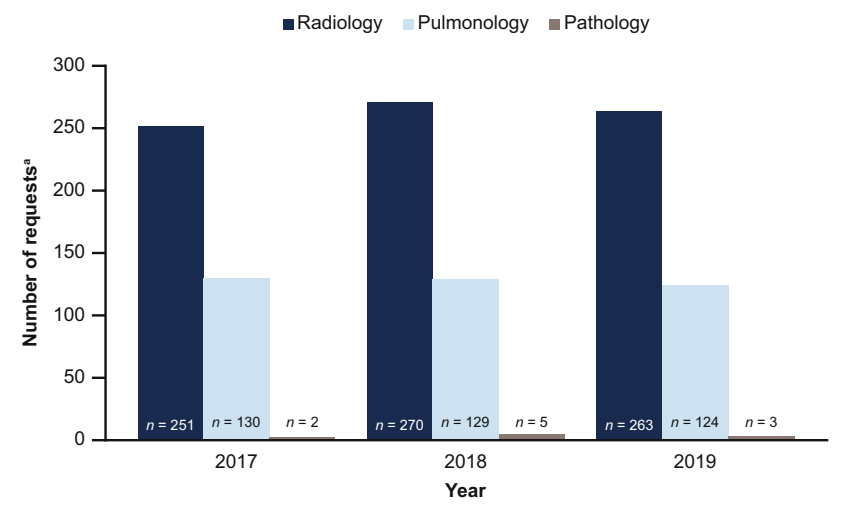

Fig. 3 Number of requests for a second opinion presented by speciality ${ }^{\text {a }}$ The sum of all requests across the specialities exceeds the total number of requests for a given year because multiple specialities could be consulted within a single request from a spoke centre

clinicians from spoke centres responded to the user survey. Due to the anonymous nature of the survey, it is not known how many different hub or spoke centres were represented and it is possible that more than one clinician from a single centre may have responded.

Of the 19 responding spoke centre clinicians (Fig. 4a), 14 (74\%) reported that the PerFECT 2.0 platform is easy/very easy to use to request a second opinion, 12 (63\%) reported that the PerFECT 2.0 platform is highly useful and $14(74 \%)$ reported that they are satisfied/very satisfied with the time taken to receive the second opinion. Seventeen (89\%) of the responding spoke centre clinicians answered positively to questions on whether the PerFECT 2.0 platform increased their confidence around diagnosing IPF/ILD, whether the platform helped them to diagnose IPF/ILD faster and whether they would recommend the platform to their colleagues. The remaining two responding spoke centre clinicians answered negatively to each of these questions; both had reported being dissatisfied/very dissatisfied with the time taken to receive the second opinion.

Of the nine hub centre clinicians (Fig. 4b), 7 (78\%) reported that the PerFECT 2.0 platform is easy/very easy to use to provide a second opinion, 4 (44\%) reported that providing a second opinion is not time consuming or a burden and $7(78 \%)$ rated the usefulness of the 
a)

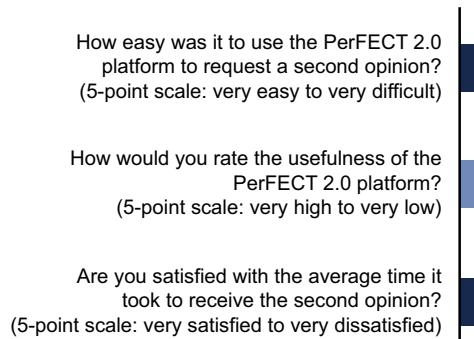
(5-point scale: very satisfied to very dissatisfied)

Did the PerFECT 2.0 platform increase your confidence around diagnosing IPF/ILD?

(Yes / No)

Do you feel the PerFECT 2.0 platform did help you to diagnose IPF/ILD faster? (Yes / No)

Would you recommend the PerFECT 2.0 platform to your colleagues? (Yes / No)

b)

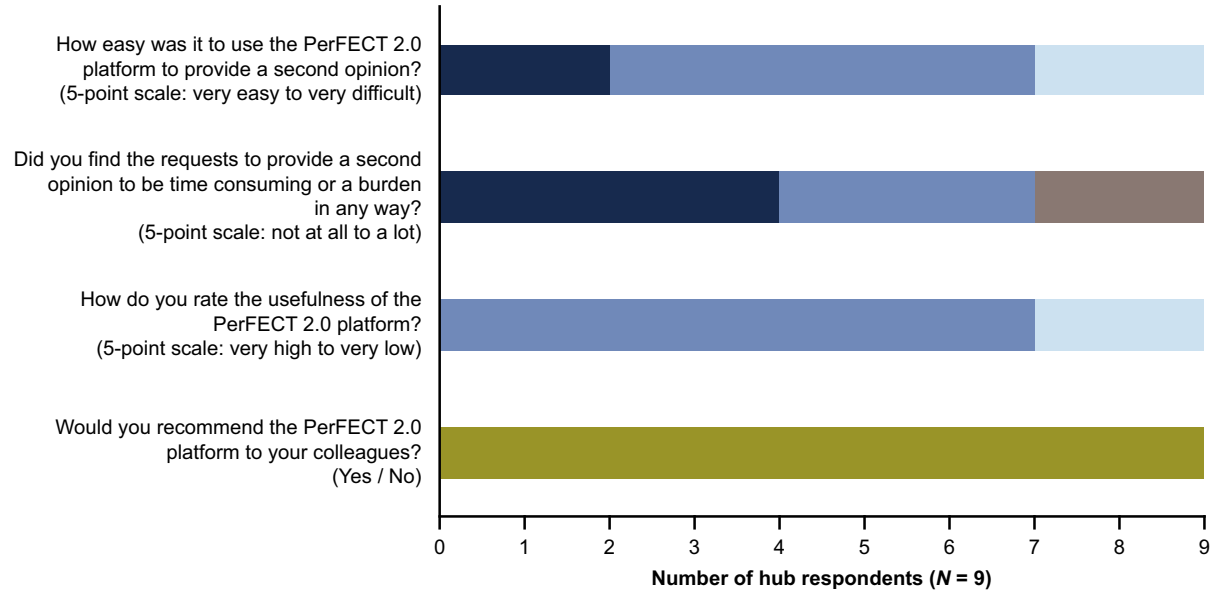

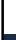

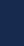
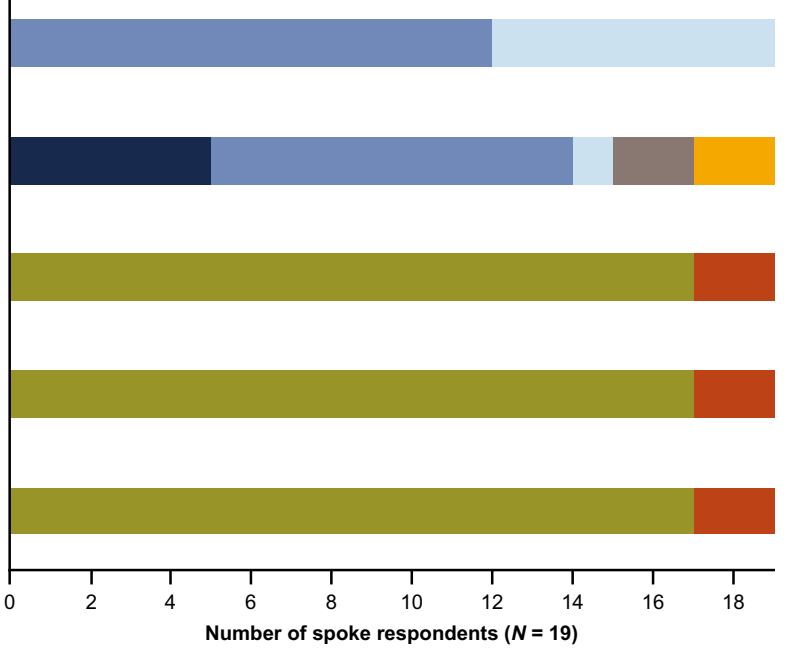

Number of spoke respondents $(N=19)$

Fig. 4 User survey results for $(\mathbf{a})$ spoke centres $(N=19)$ and $(\mathbf{b})$ hub centres $(N=9)$. ILD interstitial lung disease, $I P F$ idiopathic pulmonary fibrosis

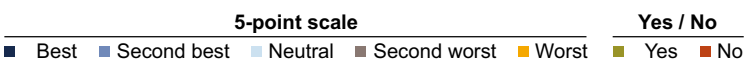

platform as high. All would recommend the platform to their colleagues.

\section{DISCUSSION}

We have described PerFECT 2.0, an Italian webbased platform that creates a network between tertiary centres with an MDT (hubs) and secondary centres (spokes) to facilitate and support the early and accurate diagnosis of IPF. Over
250 requests for a second opinion were submitted annually by spoke centres in 2017, 2018 and 2019. The vast majority of these requests received a response from hub centres. The second opinions were received quickly, with an overall mean response time (across all hub centres and years) of 15.7 days, although the mean response time varied widely among hub centres, and in 2019 ranged from 2.6 to 26.5 days. In the two most active hubs, the mean response times in 2019 were 14.4 and 
7.3 days, suggesting that second opinions could feasibly be returned using the platform in around 2 weeks or less. The user survey findings suggest that the majority of spoke centre clinicians find the platform easy to use, highly useful and satisfactory in terms of the time taken to receive the second opinion, and that the majority of hub centre clinicians find the platform easy to use to provide a second opinion.

Approaches to facilitate and support the early and accurate diagnosis of IPF are important because delays in diagnosis have been highlighted as an area of inequality and unmet need among patients with IPF, and led to a call to action put forward in 2014 as part of the European IPF Patient Charter [16]. For example, in a qualitative European survey of 45 patients with IPF, $87 \%$ of patients reported that diagnosis took over 1 year [14]. Furthermore, in an online survey of 600 patients with IPF or nonIPF ILD, the median time from symptom onset to final diagnosis was 7 months (range 0-252 months), with $43 \%$ waiting over 1 year for a diagnosis and $19 \%$ of patients waiting over 3 years for a diagnosis [19]. Similarly, results from an online survey of 1448 patients with pulmonary fibrosis and their caregivers found that $54.6 \%$ of patients had experienced a delay of at least 1 year between experiencing breathlessness and receiving a diagnosis of pulmonary fibrosis [17].

The PerFECT 2.0 platform provides an approach that addresses the barrier of limited access to specialist centres with an MDT. Access to a specialist MDT is a key part of increasing diagnostic accuracy [18], with the latest update to the IPF diagnostic guidelines providing a conditional recommendation supporting the use of multidisciplinary discussions (MDDs) as part of diagnosis [2]. However, some centres do not have access to their own MDT, and referral of patients to specialist centres with these capabilities can be affected by waiting times and geographical barriers [16]. A lack of specialists and a lack of access to specialist clinics was perceived to be a critical reason for delayed diagnosis among the patient advocacy groups consulted during development of the European IPF Patient Charter [16]. This perception is supported by findings from a prospective cohort study of 129 patients with IPF, where the median delay between onset of symptoms and evaluation at a tertiary care centre was 2.2 years [15]. A key benefit of the PerFECT 2.0 platform is to ensure quick and easy access to second opinions and specialist knowledge at the hub centres. The platform can help to eliminate delays associated with patients having to wait for an appointment at a hub centre, and can also eliminate the need to travel to these centres to receive a diagnosis or second opinion for patients living in remote areas with poor access to hub centres.

A further potential benefit of the PerFECT 2.0 platform is the ability to increase education and confidence among spoke centres in making an independent diagnosis. The European IPF Patient Group Charter highlighted a perceived lack of familiarity of IPF among some pulmonologists, and reported misconceptions about the diagnosis of IPF and a lack of awareness of diagnostic guidelines [16]. Misdiagnosis is common among patients with IPF and is a further source of delays in diagnosis. In one survey of 600 people with ILD, more than half of patients with IPF or non-IPF ILD had experienced at least one misdiagnosis [19]. It is clear that lack of knowledge about ILD and a lack of MDT support can lead to misdiagnosis and delays in accurate diagnosis. Increasing awareness of IPF among spoke centres is key to overcoming this barrier, but is made more difficult by the rare nature of IPF, which means that it is hard to gain experience and improve skills in smaller centres. The PerFECT 2.0 platform can help to train spoke centres to make autonomous diagnosis and treatment decisions in patients with IPF. The second opinions received from hub centres can help in providing practical training to spoke centres, and the case library gives further opportunities for spoke centres to increase their knowledge base and confidence in diagnosing complex cases. In the user survey, the majority of spoke centres answered positively to questions on whether the platform increased their confidence around diagnosing IPF/ILD and helped them to make diagnoses faster. For those spoke centres that do not have their own MDT, the PerFECT 2.0 platform can 
provide ongoing support and access to ILD specialists at hub centres.

The PerFECT 2.0 platform is not the only example of technology being utilised in the diagnosis of ILDs. In Japan, clinical, radiological and pathological data from 524 patients across 39 centres were entered into a cloud-based integrated database of idiopathic interstitial pneumonia [20]. For the 465 cases with sufficient data, web-based MDDs were conducted via video conference. Approximately half $(47 \%)$ of the diagnoses were subsequently changed after the MDDs, and survival analyses comparing original diagnoses with those after the MDDs revealed that prognostic discrimination between idiopathic interstitial pneumonia was improved by the MDDs. Another example is the STARLINER study, which investigated disease behaviour in the time periods directly before and after diagnosis in patients with suspected ILD. This study utilised a digital collaboration platform, which allowed virtual MDDs to take place between secondary and tertiary centres $[13,21]$. Unlike the PerFECT 2.0 platform, both of these examples involved virtual MDDs being hosted live on the digital platform. In contrast, the PerFECT 2.0 platform involves spoke centres submitting requests for a second opinion from a specific speciality at a hub centre (pulmonology, radiology and/or pathology), with responses returned via the platform without any live discussions. However, all three platforms are examples of how technology can facilitate the diagnosis of ILDs, laying the foundation for increased use of these technologies in the future.

Recently, the COVID-19 pandemic has made it more difficult for patients to visit tertiary centres with an MDT face-to-face, and favours the development of digital technologies to enhance telemedicine services. The metrics and user survey findings for the PerFECT 2.0 platform show that digital technology and systems to gain online access to a specialist opinion to facilitate diagnoses can be easily applied in the ILD setting. The ability of the clinicians at hub centres to access documentation online, including HRCT images, pathological samples and clinical data, is of particular benefit.
A limitation of the PerFECT 2.0 platform is the absence of direct patient contact from the hub centres, which can put the focus on the disease rather than the patient; nonetheless, direct contact between the patient and clinician is maintained at the secondary centre. Also, the clinical data available to the hub centres, although reasonably wide ranging, are limited by the questions asked by the platform. A further limitation is that the number of patients undergoing lung biopsy is not captured unless a second opinion is requested. The number of requests for a pathology second opinion via the platform between 2017 and 2019 was low in comparison to the number of pulmonology and radiology requests; however, it is not possible to determine whether biopsies are being performed but not sent for a second opinion, for example, due to the need to ship the samples to hub centres, or whether the access to pulmonology or radiology second opinions via the platform is reducing the need for a biopsy if a working diagnosis can be formed without one. Another limitation of the platform is that radiologists at hub centres may experience issues with the quality of the uploaded HRCT image, leading to potential delays in obtaining an accurate diagnosis. As found in the user survey, the hub centres can sometimes take a longer time than is satisfactory to return a second opinion. In addition, administrative aspects of the platform can cause inconvenience, particularly around contracting, because each hub and spoke centre is required to renew their contract every year, which can be subject to delays during which time the centres cannot access the platform. The entrusting of managing sensitive data to a third party can also be problematic at some hospitals. Regarding the limitations of our survey data, it should be noted that only a small proportion of spoke and hub users responded to the survey, most likely due to the COVID-19 situation in Italy during the time that the survey was open to responses. Finally, as the PerFECT 2.0 platform has only been utilised in Italy, it is not possible to directly comment on how feasible it would be to use the platform to create a network of hub and spoke centres in countries with different healthcare systems. 
In conclusion, the PerFECT 2.0 web-based platform has created a network that enables secondary centres to gain quick and easy access to a second opinion from a tertiary centre with an MDT through online evaluation of uploaded anonymised documentation (HRCT images, pathological samples and clinical data), thereby facilitating and supporting the early and accurate diagnosis of IPF. The majority of hub and spoke centre users find the platform easy to use and would recommend it to colleagues.

\section{ACKNOWLEDGEMENTS}

The authors would like to thank Klaus-Uwe Kirchgässler (F. Hoffman-La Roche, Ltd.), Michele Davì (Roche S.p.A.) and Luca Morelli (DOT Tech srl) for their ongoing support of this platform. The authors would also like to thank the hub and spoke centres that have participated in the platform, and the therapy-area experts who supported the development of the platform.

Funding. The PerFECT 2.0 platform is sponsored by Roche S.p.A. (Monza, Italy). The Pulmonary Therapy Rapid Service Fee was funded by F. Hoffmann-La Roche, Ltd.

Authorship. All named authors meet the International Committee of Medical Journal Editors (ICMJE) criteria for authorship of this article, take responsibility for the integrity of the work as a whole and have given their approval for this final version to be published.

Medical Writing, Editorial and Other Assistance. Medical writing support was provided by Catherine Stanton and Leigh Clements, PhD, of CMC AFFINITY, McCann Health Medical Communications, funded by F. Hoffmann-La Roche, Ltd.

Disclosures. Carlo Vancheri, Donato Lacedonia, Alessandro Sanduzzi, Gaetano Rea and Alberto Pesci have received grants and speaker and personal fees from Boehringer Ingelheim and F. Hoffmann-La Roche, Ltd. Monica Bengus and Sonja A. Rohner are employees and shareholders of F. Hoffmann-La Roche, Ltd. Laura Bianchino and Alessandra Ghirardini are employees of Roche S.p.A. Maria G. Cagnazzo and Mercedes Pasquali have no conflict of interest relevant to the current publication. Sebastiano E. Torrisi has received personal fees and grants from Boehringer Ingelheim and F. Hoffmann-La Roche, Ltd.

Compliance with Ethics Guidelines. The platform is constantly supervised (upon upgrades or updates) by a third-party company (external Data Protection Officer) to ensure compliance with European Union Regulation 2016/679 (General Data Protection Regulation) and The Italian Data Protection Authority (for processing of sensitive data). No ethical approval was required. Roche S.p.A. does not have access to personal information collected by the platform and only receives operational metrics data on the platform's use.

Data Availability. Qualified researchers may request access to individual patient-level data through the clinical study data request platform (https://vivli.org/). Further details on Roche's criteria for eligible studies are available here (https://vivli.org/members/ourmembers/). For further details on Roche's Global Policy on the Sharing of Clinical Information and how to request access to related clinical study documents, see here (https://www.roche.com/ research_and_development/who_we_are_how_ we_work/clinical_trials/our_commitment_to_ data_sharing.htm).

Open Access. This article is licensed under a Creative Commons Attribution-NonCommercial 4.0 International License, which permits any non-commercial use, sharing, adaptation, distribution and reproduction in any medium or format, as long as you give appropriate credit to the original author(s) and the source, provide a link to the Creative Commons licence, and indicate if changes were made. The images or other third party material in this article are included in the article's Creative Commons licence, unless indicated otherwise in a credit line to the material. If material is not included 
in the article's Creative Commons licence and your intended use is not permitted by statutory regulation or exceeds the permitted use, you will need to obtain permission directly from the copyright holder. To view a copy of this licence, visit http://creativecommons.org/licenses/by$\mathrm{nc} / 4.0 /$.

\section{REFERENCES}

1. Ley B, Collard HR, King TE Jr. Clinical course and prediction of survival in idiopathic pulmonary fibrosis. Am J Respir Crit Care Med. 2011;183: 431-40.

2. Raghu G, Remy-Jardin M, Myers JL, et al. Diagnosis of idiopathic pulmonary fibrosis. An official ATS/ ERS/JRS/ALAT Clinical Practice Guideline. Am J Respir Crit Care Med. 2018;198:e44-68.

3. Hutchinson J, Fogarty A, Hubbard R, McKeever T. Global incidence and mortality of idiopathic pulmonary fibrosis: a systematic review. Eur Respir J. 2015;46:795-806.

4. Food and Drug Administration. Highlights of Prescribing Information-OFEV ${ }^{\circledR}$. 2020. https://www. accessdata.fda.gov/drugsatfda_docs/label/2020/ 205832s013lbl.pdf. Accessed 25 Jan 2021.

5. Food and Drug Administration. Highlights of Prescribing Information-ESBRIET ${ }^{\circledR}$. 2019. https:// www.accessdata.fda.gov/drugsatfda_docs/label/ 2019/022535s012,208780s002lbl.pdf. Accessed 25 Jan 2021.

6. European Medicines Agency. Summary of Product Characteristics-Esbriet (pirfenidone). 2020. https://www.ema.europa.eu/en/documents/ product-information/esbriet-epar-productinformation_en.pdf. Accessed 25 Jan 2021.

7. European Medicines Agency. Summary of Product Characteristics-Ofev (nintedanib). 2020. https:// www.ema.europa.eu/en/documents/productinformation/ofev-epar-product-information_en. pdf. Accessed 25 Jan 2021.

8. Richeldi L, du Bois RM, Raghu G, et al. Efficacy and safety of nintedanib in idiopathic pulmonary fibrosis. N Engl J Med. 2014;370:2071-82.

9. King TE Jr, Bradford WZ, Castro-Bernardini S, et al. A phase 3 trial of pirfenidone in patients with idiopathic pulmonary fibrosis. $\mathrm{N}$ Engl $\mathrm{J}$ Med. 2014;370:2083-92.
10. Noble PW, Albera C, Bradford WZ, et al. Pirfenidone in patients with idiopathic pulmonary fibrosis (CAPACITY): two randomised trials. Lancet. 2011;377:1760-9.

11. Cordier JF, Cottin V. Neglected evidence in idiopathic pulmonary fibrosis: from history to earlier diagnosis. Eur Respir J. 2013;42:916-23.

12. Wijsenbeek MS, Bendstrup E, Valenzuela C, et al. Interim analysis of patients with ILD enrolled in the STARLINER study. Eur Respir J. 2019;54(Suppl 63): PA1335.

13. Wijsenbeek MS, Bendstrup E, Valenzuela C, et al. Primary results from the STARLINER study of disease behaviour in suspected ILD. Eur Respir J. 2020;56:1794.

14. Russell AM, Ripamonti E, Vancheri C. Qualitative European survey of patients with idiopathic pulmonary fibrosis: patients' perspectives of the disease and treatment. BMC Pulm Med. 2016;16:10.

15. Lamas DJ, Kawut SM, Bagiella E, Philip N, Arcasoy SM, Lederer DJ. Delayed access and survival in idiopathic pulmonary fibrosis: a cohort study. Am J Respir Crit Care Med. 2011;184:842-7.

16. Bonella F, Wijsenbeek M, Molina-Molina M, et al. European IPF Patient Charter: unmet needs and a call to action for healthcare policymakers. Eur Respir J. 2016;47:597-606.

17. Collard HR, Tino G, Noble PW, et al. Patient experiences with pulmonary fibrosis. Respir Med. 2007;101:1350-4.

18. Chaudhuri N, Spencer L, Greaves M, Bishop P, Chaturvedi A, Leonard C. A review of the multidisciplinary diagnosis of interstitial lung diseases: a retrospective analysis in a single UK specialist centre. J Clin Med. 2016;5:66.

19. Cosgrove GP, Bianchi P, Danese S, Lederer DJ. Barriers to timely diagnosis of interstitial lung disease in the real world: the INTENSITY survey. BMC Pulm Med. 2018;18:9.

20. Fujisawa T, Mori K, Mikamo M, et al. Nationwide cloud-based integrated database of idiopathic interstitial pneumonias for multidisciplinary discussion. Eur Respir J. 2019;53:1802243.

21. Wijsenbeek M, Bendstrup E, Valenzuela C, et al. Design of a study assessing disease behaviour during the peri-diagnostic period in patients with interstitial lung disease: the STARLINER study. Adv Ther. 2019;36:232-43. 